\title{
PRICING MULTI-ASSET FINANCIAL DERIVATIVES WITH TIME-DEPENDENT PARAMETERS-LIE ALGEBRAIC APPROACH
}

\author{
C. F. LO and C. H. HUI
}

Received 31 October 2001

\begin{abstract}
We present a Lie algebraic technique for the valuation of multi-asset financial derivatives with time-dependent parameters. Exploiting the dynamical symmetry of the pricing partial differential equations of the financial derivatives, the new method enables us to derive analytical closed-form pricing formulae very straightforwardly. We believe that this new approach will provide an efficient and easy-to-use method for the valuation of financial derivatives.
\end{abstract}

2000 Mathematics Subject Classification: 91B28, 70G65, 35K15.

1. Introduction. The Lie algebraic method is introduced by Lo and Hui [8] to the field of finance for the pricing of single-asset financial derivatives with time-dependent model parameters. This new method is based upon the Wei-Norman theorem (Wei and Norman [12]) and has never been used in the field of finance. It is very simple and has been successfully applied to tackle time-dependent Schrödinger equation associated with generalized quantum time-dependent oscillators (Lo [2, 3], Ng and Lo [10], and Lo and Wong [9]) as well as the Fokker-Planck equation (Lo [4, 5, 6, 7]). Exploiting the well-defined algebraic structures of the pricing partial differential equations, analytical closed-form pricing formulae can be derived for financial derivatives with time-dependent parameters. For demonstration, we have applied the Lie algebraic approach to value European options for the constant elasticity of variance (CEV) process and corporate discount bonds with default risk. In this paper, we will extend the Lie algebraic approach to the valuation of financial derivatives involving multi-assets and stochastic interest rate, for example, multi-asset options with and without stochastic short-term interest rate. In the valuation of these financial derivatives, the value of each of the underlying assets is assumed to follow the usual lognormal diffusion process

$$
\frac{d S_{i}}{S_{i}}=\mu_{i}(t) d t+\sigma_{i}(t) d Z_{i}, \quad 1 \leq i \leq N,
$$

where $\mu_{i}(t)$ and $\sigma_{i}(t)$ are the drift and volatility of the value of asset $i$, respectively. The dynamics of the short-term interest rate $r$ is drawn from the term structure model (Vasicek [11])

$$
d r=\kappa(t)[\theta(t)-r] d t+\sigma_{r}(t) d Z_{r},
$$


where the short-term interest rate is mean-reverting to long-term mean $\theta(t)$ at speed $\kappa(t)$ and $\sigma_{r}(t)$ is the volatility of $r$. The Wiener processes $d Z_{r}$ and $d Z_{i}$ are correlated with

$$
d Z_{i} d Z_{r}=\rho_{i r}(t) d t, \quad d Z_{i} d Z_{j}=\rho_{i j}(t) d t,
$$

where $\rho_{i r}(t)$ and $\rho_{i j}(t)$ are the correlation coefficients, and we must necessarily have $\rho_{r r}(t)=\rho_{i i}(t)=1,-1<\rho_{i r}(t)=\rho_{r i}(t)<1$, and $-1<\rho_{i j}(t)=\rho_{j i}(t)<1$ for $1 \leq$ $i, j \leq N$. It has been pointed out that such a pricing problem is rather formidable and defies the conventional approach for the single-asset Black-Scholes model with timedependent parameters (Bos and Ware [1]). Nevertheless, within the framework of the Lie algebraic approach, the generalization is very simple and straightforward.

This paper is organized as follows. Section 2 outlines the Wei-Norman theorem and its applications. Section 3 applies the Lie algebraic technique to the valuation problem of multi-asset options in which the short-term interest rate is not treated as a stochastic variable. Section 4 studies the pricing of multi-asset options with stochastic short-term interest rate using the new valuation approach. Finally, Section 5 briefly summaries and concludes the paper.

2. Wei-Norman theorem. Consider the linear operator differential equation of the first order

$$
\frac{d U(t)}{d t}=H(t) U(t) ; \quad U(0)=1
$$

where $H$ and $U$ are both time-dependent linear operators in a Banach space or a finitedimensional space. According to the Wei-Norman theorem (Wei and Norman [12]), if the operator $H$ can be expressed as

$$
H(t)=\sum_{n=1}^{N} a_{n}(t) L_{n},
$$

where $a_{n}$ 's are scalar functions of time and $L_{n}$ are the generators of an $N$-dimensional solvable Lie algebra or the real split 3-dimensional simple Lie algebra, then the operator $U$ can be expressed as

$$
U(t)=\prod_{n=1}^{N} \exp \left[g_{n}(t) L_{n}\right] .
$$

Here the $g_{n}$ 's are time-dependent scalar functions to be determined. To find the $g_{n}$ 's, we simply substitute (2.2) and (2.3) into (2.1) and compare the two sides term by term to obtain a set of coupled nonlinear differential equations

$$
\frac{d g_{n}(t)}{d t}=\sum_{m=1}^{N} \eta_{n m} a_{m}(t), \quad g_{n}(0)=0,
$$

where $\eta_{n m}$ are nonlinear functions of $g_{n}$ 's. Thus, we have reduced the linear operator differential equation (2.1) to a set of coupled nonlinear differential equations of scalar functions (2.4). 
For illustration, we consider the special case that the generators $L_{n}$ 's form the Heisenberg-Weyl Lie algebra defined by the commutation relations

$$
\left[L_{1}, L_{2}\right]=L_{3}, \quad\left[L_{1}, L_{3}\right]=\left[L_{2}, L_{3}\right]=0
$$

Then, $H$ is given by

$$
H(t)=a_{1}(t) L_{1}+a_{2}(t) L_{2}+a_{3}(t) L_{3}
$$

According to the Wei-Norman theorem, $U(t)$ can be expressed as

$$
U(t)=\exp \left[g_{1}(t) L_{1}\right] \cdot \exp \left[g_{2}(t) L_{2}\right] \cdot \exp \left[g_{3}(t) L_{3}\right]
$$

By differentiation, we obtain

$$
\begin{aligned}
\frac{d U(t)}{d t} U(t)^{-1}= & \frac{d g_{1}(t)}{d t} L_{1}+\frac{d g_{2}(t)}{d t} \exp \left[g_{1}(t) L_{1}\right] L_{2} \exp \left[-g_{1}(t) L_{1}\right] \\
& +\frac{d g_{3}(t)}{d t} \exp \left[g_{1}(t) L_{1}\right] \exp \left[g_{2}(t) L_{2}\right] L_{3} \exp \left[-g_{2}(t) L_{2}\right] \exp \left[-g_{1}(t) L_{1}\right] \\
= & \frac{d g_{1}(t)}{d t} L_{1}+\frac{d g_{2}(t)}{d t} L_{2}+\left[\frac{d g_{3}(t)}{d t}+g_{1}(t) \frac{d g_{2}(t)}{d t}\right] L_{3} .
\end{aligned}
$$

Comparing (2.6) and (2.8) yields a set of three coupled nonlinear differential equations

$$
\begin{gathered}
\frac{d g_{1}(t)}{d t}=a_{1}(t), \quad \frac{d g_{2}(t)}{d t}=a_{2}(t), \\
\frac{d g_{3}(t)}{d t}+g_{1}(t) \frac{d g_{2}(t)}{d t}=a_{3}(t) .
\end{gathered}
$$

It is not difficult to show that the set of differential equations can be easily solved by quadrature

$$
\begin{aligned}
& g_{1}(t)=\int_{0}^{t} d \tau a_{1}(\tau) \\
& g_{2}(t)=\int_{0}^{t} d \tau a_{2}(\tau) \\
& g_{3}(t)=\int_{0}^{t} d \tau\left[a_{3}(\tau)-a_{2}(\tau) g_{1}(\tau)\right]
\end{aligned}
$$

As a result, the operator $U(t)$ is thus determined.

3. Multi-asset European options. The fair price $P\left(S_{1}, S_{2}, \ldots, S_{n}, t\right)$ of a multi-asset European option with time-dependent parameters can be determined by solving the multi-asset generalization of the Black-Scholes equation

$$
\frac{\partial P}{\partial t}=\frac{1}{2} \sum_{i, j=1}^{n} \sigma_{i}(t) \sigma_{j}(t) \rho_{i j}(t) S_{i} S_{j} \frac{\partial^{2} P}{\partial S_{i} \partial S_{j}}+\sum_{i=1}^{n}\left[r(t)-d_{i}(t)\right] S_{i} \frac{\partial P}{\partial S_{i}}-r(t) P,
$$


where $t$ is the time to maturity. Introducing the new variables $x_{i}=\ln \left(S_{i}\right)$, the pricing equation is simplified to

$$
\begin{aligned}
\frac{\partial P}{\partial t}= & \frac{1}{2} \sum_{i, j=1}^{n} \sigma_{i}(t) \sigma_{j}(t) \rho_{i j}(t) \frac{\partial^{2} P}{\partial x_{i} \partial x_{j}} \\
& +\sum_{i=1}^{n}\left[r(t)-d_{i}(t)-\frac{\sigma_{i}(t)^{2}}{2}\right] \frac{\partial P}{\partial x_{i}}-r(t) P \\
\equiv & {[H(t)-r(t)] P . }
\end{aligned}
$$

It is obvious that the operator $H(t)$ can be rewritten as follows:

$$
H(t)=\sum_{i, j=1}^{n} A_{i j}(t) \hat{L}_{i j}+\sum_{i=1}^{n} B_{i}(t) \hat{D}_{i}
$$

where

$$
\begin{gathered}
\hat{L}_{i j}=\frac{\partial^{2}}{\partial x_{i} \partial x_{j}}, \quad \hat{D}_{i}=\frac{\partial}{\partial x_{i}} \\
A_{i j}(t)=\frac{1}{2} \sigma_{i}(t) \sigma_{j}(t) \rho_{i j}(t), \quad B_{i}(t)=r(t)-d_{i}(t)-\frac{\sigma_{i}^{2}}{2} .
\end{gathered}
$$

The operators $L_{i j}$ and $D_{i}$ form a solvable algebra; in fact, they all commute. We may now define the evolution operator $U(t, 0)$ such that

$$
P\left(x_{1}, x_{2}, \ldots, x_{n}, t\right)=\exp \left[-\int_{0}^{t} r\left(t^{\prime}\right) d t^{\prime}\right] \cdot U(t, 0) P\left(x_{1}, x_{2}, \ldots, x_{n}, 0\right) .
$$

Inserting (3.5) into (3.2) yields the evolution equation

$$
\frac{\partial}{\partial t} U(t, 0)=H(t) U(t, 0), \quad U(0,0)=1 .
$$

Since the operators $L_{i j}$ and $D_{i}$ all commute with each other, the Wei-Norman theorem states that the evolution operator $U(t, 0)$ can be expressed in the form (Wei and Norman [12])

$$
U(t, 0)=\prod_{i=1}^{n} \exp \left[b_{i}(t) \hat{D}_{i}\right] \cdot \prod_{i, j=1}^{n} \exp \left[a_{i j}(t) \hat{L}_{i j}\right]
$$

where the coefficients $a_{i j}(t)$ and $b_{i}(t)$ are simply given by

$$
\begin{aligned}
a_{i j}(t) & =\frac{1}{2} \int_{0}^{t} \sigma_{i}\left(t^{\prime}\right) \sigma_{j}\left(t^{\prime}\right) \rho_{i j}\left(t^{\prime}\right) d t^{\prime}, \\
b_{i}(t) & =\int_{0}^{t}\left[r\left(t^{\prime}\right)-d_{i}\left(t^{\prime}\right)-\frac{\sigma_{i}\left(t^{\prime}\right)^{2}}{2}\right] d t^{\prime} .
\end{aligned}
$$

Hence, we have found an exact form of the time evolution operator $U(t, 0)$. 
We define $\mathbf{a}(t)$ as the $n \times n$ matrix whose elements are given by $a_{i j}(t)$, and $\mathbf{a}^{-1}(t)$ as its inverse. Then, it is not difficult to show that

$$
\begin{aligned}
P\left(x_{1}, x_{2}, \ldots, x_{n}, t\right)= & \int_{-\infty}^{\infty} d y_{1} \int_{-\infty}^{\infty} d y_{2} \ldots \int_{-\infty}^{\infty} d y_{n} P\left(y_{1}, y_{2}, \ldots, y_{n}, 0\right) \\
& \times K\left(x_{1}, x_{2}, \ldots, x_{n}, t ; y_{1}, y_{2}, \ldots, y_{n}, 0\right),
\end{aligned}
$$

where

$$
\begin{aligned}
K\left(x_{1}, x_{2}, \ldots, x_{n}, t ; y_{1}, y_{2}, \ldots, y_{n}, 0\right) \\
=\frac{1}{\sqrt{(4 \pi)^{n} \operatorname{det}(\mathbf{a})}} \exp \left[-\int_{0}^{t} r\left(t^{\prime}\right) d t^{\prime}\right] \\
\quad \times \exp \left\{-\frac{1}{4} \sum_{i, j=1}^{n}\left(x_{i}-y_{i}+b_{i}\right)\left(\mathbf{a}^{-1}\right)_{i j}\left(x_{j}-y_{j}+b_{j}\right)\right\}
\end{aligned}
$$

is the propagator of the pricing equation in (3.2). With $n=1$, we will recover the well-known result of single-asset option pricing.

4. Multi-asset European options with stochastic interest rate. In the presence of stochastic short-term interest rate, the price $P\left(S_{1}, S_{2}, \ldots, S_{n}, r, t\right)$ of a multi-asset European option obeys the partial differential equation

$$
\begin{aligned}
\frac{\partial P}{\partial t}= & \frac{1}{2} \sum_{i, j=1}^{n} \sigma_{i}(t) \sigma_{j}(t) \rho_{i j}(t) S_{i} S_{j} \frac{\partial^{2} P}{\partial S_{i} \partial S_{j}} \\
& +\frac{1}{2} \sigma_{r}(t)^{2} \frac{\partial^{2} P}{\partial r^{2}}+\sum_{i=1}^{n} \sigma_{i}(t) \sigma_{r}(t) \rho_{i r}(t) S_{i} \frac{\partial^{2} P}{\partial S_{i} \partial r} \\
& +\sum_{i=1}^{n}\left[r-d_{i}(t)\right] S_{i} \frac{\partial P}{\partial S_{i}}+\kappa(t)[\theta(t)-r] \frac{\partial P}{\partial r}-r P \\
= & \frac{1}{2} \sum_{i, j=1}^{n} \sigma_{i}(t) \sigma_{j}(t) \rho_{i j}(t) \frac{\partial^{2} P}{\partial x_{i} \partial x_{j}} \\
& +\frac{1}{2} \sigma_{r}(t)^{2} \frac{\partial^{2} P}{\partial r^{2}}+\sum_{i=1}^{n} \sigma_{i}(t) \sigma_{r}(t) \rho_{i r}(t) \frac{\partial^{2} P}{\partial x_{i} \partial r} \\
& +\sum_{i=1}^{n}\left[r-d_{i}(t)-\frac{1}{2} \sigma_{i}(t)^{2}\right] \frac{\partial P}{\partial x_{i}}+\kappa(t)[\theta(t)-r] \frac{\partial P}{\partial r}-r P
\end{aligned}
$$

where $x_{i}=\ln \left(S_{i}\right)$ and $t$ is the time to maturity. To solve this partial differential equation, we first define the evolution operator $U(t, 0) \equiv U_{0}(t, 0) U_{I}(t, 0)$ such that

$$
\begin{aligned}
P\left(x_{1}, x_{2}, \ldots, x_{n}, r, t\right) & =U(t, 0) P\left(x_{1}, x_{2}, \ldots, x_{n}, r, 0\right) \\
& =U_{0}(t, 0) U_{I}(t, 0) P\left(x_{1}, x_{2}, \ldots, x_{n}, r, 0\right) .
\end{aligned}
$$

Inserting (4.2) into (4.1) yields the evolution equations

$$
\begin{array}{ll}
H_{0}(t) U_{0}(t, 0)=\frac{\partial}{\partial t} U_{0}(t, 0), & U_{0}(0,0)=1, \\
H_{I}(t) U_{I}(t, 0)=\frac{\partial}{\partial t} U_{I}(t, 0), & U_{I}(0,0)=1,
\end{array}
$$


where

$$
\begin{aligned}
H_{0}(t)= & \frac{1}{2} \sum_{i, j=1}^{n} \sigma_{i}(t) \sigma_{j}(t) \rho_{i j}(t) \frac{\partial^{2}}{\partial x_{i} \partial x_{j}}+\frac{1}{2} \sigma_{r}(t)^{2} \frac{\partial^{2}}{\partial r^{2}} \\
& +\sum_{i=1}^{n} \sigma_{i}(t) \sigma_{r}(t) \rho_{i r}(t) \frac{\partial^{2}}{\partial x_{i} \partial r}+\sum_{i=1}^{n} r \frac{\partial}{\partial x_{i}}-\kappa(t) r \frac{\partial}{\partial r}
\end{aligned}
$$

and $H_{I} \equiv U_{0}(t, 0)^{-1}\left[H(t)-H_{0}(t)\right] U_{0}(t, 0)$. It is not difficult to show that the operator $H_{0}(t)$ can be rewritten in the following form:

$$
H_{0}(t)=\sum_{i, j=1}^{n} A_{i j}(t) \hat{L}_{i j}+\sum_{i=1}^{n} E_{i}(t) \hat{D}_{i}+\sum_{i=1}^{n} F_{i}(t) \hat{M}_{i}+B_{1} \hat{J}_{1}+B_{2} \hat{J}_{2},
$$

where

$$
\begin{gathered}
\hat{L}_{i j}=\frac{\partial^{2}}{\partial x_{i} \partial x_{j}}, \quad \hat{D}_{i}=r \frac{\partial}{\partial x_{i}}, \quad \hat{M}_{i}=\frac{\partial^{2}}{\partial x_{i} \partial r}, \\
\hat{J}_{2}=\frac{\partial^{2}}{\partial r^{2}}, \quad \hat{J}_{1}=r \frac{\partial}{\partial r}, \\
A_{i j}(t)=\frac{1}{2} \sigma_{i}(t) \sigma_{j}(t) \rho_{i j}(t), \quad B_{1}(t)=-\kappa(t), \quad B_{2}(t)=\frac{1}{2} \sigma_{r}(t)^{2}, \\
E_{i}(t)=1, \quad F_{i}(t)=\sigma_{i}(t) \sigma_{r}(t) \rho_{i r}(t) .
\end{gathered}
$$

The operators $\hat{L}_{i j}, \hat{D}_{i}, \hat{M}_{i}$, and $\hat{J}_{i}$ form a solvable Lie algebra

$$
\begin{gathered}
{\left[\hat{L}_{i j}, \hat{L}_{k l}\right]=\left[\hat{L}_{i j}, \hat{D}_{k}\right]=\left[\hat{L}_{i j}, \hat{M}_{k}\right]=\left[\hat{L}_{i j}, \hat{J}_{1}\right]=\left[\hat{L}_{i j}, \hat{J}_{2}\right]=\left[\hat{M}_{i}, \hat{J}_{2}\right]=0,} \\
{\left[\hat{D}_{i}, \hat{M}_{j}\right]=-\hat{L}_{i j}, \quad\left[\hat{D}_{i}, \hat{J}_{1}\right]=-\hat{D}_{i}, \quad\left[\hat{D}_{i}, \hat{J}_{2}\right]=-2 \hat{M}_{i},} \\
{\left[\hat{M}_{i}, \hat{J}_{1}\right]=\hat{M}_{i}, \quad\left[\hat{J}_{1}, \hat{J}_{2}\right]=-2 \hat{J}_{2},}
\end{gathered}
$$

where $i, j, k, l=1,2,3, \ldots, n$. According to the Wei-Norman theorem (Wei and Norman [12]), the evolution operator $U_{0}(t, 0)$ can be expressed in the form

$$
\begin{aligned}
U_{0}(t, 0)= & \exp \left[\sum_{i=1}^{n} b_{i}(t) \hat{D}_{i}\right] \exp \left[\sum_{i, j=1}^{n} a_{i j}(t) \hat{L}_{i j}\right] \exp \left[c_{2}(t) \hat{J}_{2}\right] \\
& \times \exp \left[\sum_{i=1}^{n} f_{i}(t) \hat{M}_{i}\right] \exp \left[c_{1}(t) \hat{J}_{1}\right]
\end{aligned}
$$

where the coefficients $a_{i j}(t), c_{i}(t), b_{i}(t)$, and $f_{i}(t)$ are to be determined. Then, by direct differentiation with respect to $t$, we obtain

$$
\begin{aligned}
\frac{\partial U_{0}(t, 0)}{\partial t} U_{0}(t, 0)^{-1}= & \sum_{i, j=1}^{n} g_{i j}(t) \hat{L}_{i j}+\sum_{i=1}^{n} h_{i}(t) \hat{D}_{i} \\
& +\sum_{i=1}^{n} p_{i}(t) \hat{M}_{i}+q_{1}(t) \hat{J}_{1}+q_{2}(t) \hat{J}_{2}
\end{aligned}
$$


with

$$
\begin{gathered}
g_{i j}(t)=\frac{\partial a_{i j}}{\partial t}-b_{j} \frac{\partial f_{i}}{\partial t}+b_{i} b_{j} \frac{\partial c_{2}}{\partial t}+b_{j}\left(2 c_{2} b_{i}-f_{i}\right) \frac{\partial c_{1}}{\partial t} \\
h_{i}(t)=\frac{\partial b_{i}}{\partial t}-b_{i} \frac{\partial c_{1}}{\partial t} \\
p_{i}(t)=\frac{\partial f_{i}}{\partial t}-2 b_{i} \frac{\partial c_{2}}{\partial t}-\left(4 c_{2} b_{i}-f_{i}\right) \frac{\partial c_{1}}{\partial t} \\
q_{1}(t)=\frac{\partial c_{1}}{\partial t}, \quad q_{2}(t)=\frac{\partial c_{2}}{\partial t}+2 c_{2} \frac{\partial c_{1}}{\partial t}
\end{gathered}
$$

Substituting (4.7), (4.10), and (4.11) into (4.3), and comparing the two sides, we, after simplification, find

$$
\begin{gathered}
c_{1}(t)=\int_{0}^{t} d t^{\prime} B_{1}\left(t^{\prime}\right), \\
c_{2}(t)=\exp \left[-2 c_{1}(t)\right] \int_{0}^{t} d t^{\prime} B_{2}\left(t^{\prime}\right) \exp \left[2 c_{1}\left(t^{\prime}\right)\right], \\
b_{i}(t)=\exp \left[c_{1}(t)\right] \int_{0}^{t} d t^{\prime} E_{i}\left(t^{\prime}\right) \exp \left[-c_{1}\left(t^{\prime}\right)\right], \\
f_{i}(t)=\exp \left[-c_{1}(t)\right] \int_{0}^{t} d t^{\prime}\left\{F_{i}\left(t^{\prime}\right)+2 B_{2}\left(t^{\prime}\right) b_{i}\left(t^{\prime}\right)\right\} \exp \left[c_{1}\left(t^{\prime}\right)\right], \\
a_{i j}(t)=\int_{0}^{t} d t^{\prime}\left\{A_{i j}\left(t^{\prime}\right)+\left[F_{i}\left(t^{\prime}\right)+B_{2}\left(t^{\prime}\right) b_{i}\left(t^{\prime}\right)\right] b_{j}\left(t^{\prime}\right)\right\} .
\end{gathered}
$$

Once the coefficients $a_{i j}(t), c_{i}(t), b_{i}(t)$, and $f_{i}(t)$ are known, the operator $U_{0}(t, 0)$ is uniquely determined.

Next, using the above explicit form of the operator $U_{0}(t, 0)$, we can obtain the exact form of the operator $H_{I}(t)$

$$
\begin{aligned}
H_{I}(t)= & \sum_{i=1}^{n}\left\{f_{i}(t)+\kappa(t) \theta(t) b_{i}(t)-\left[d_{i}(t)+\frac{1}{2} \sigma_{i}(t)^{2}\right]\right\} \frac{\partial}{\partial x_{i}} \\
& +\left\{\kappa(t) \theta(t)+2 c_{2}(t)\right\} \exp \left[c_{1}(t)\right] \frac{\partial}{\partial r}-r \exp \left[-c_{1}(t)\right] .
\end{aligned}
$$

It is easy to see that the operator $U_{I}(t, 0)$ can be expressed in the form

$$
U_{I}(t, 0)=\exp \left[\sum_{i=1}^{n} \xi_{i}(t) \frac{\partial}{\partial x_{i}}\right] u(t, 0)
$$

where

$$
\xi_{i}(t)=\int_{0}^{t} d t^{\prime}\left\{f_{i}\left(t^{\prime}\right)+\kappa\left(t^{\prime}\right) \theta\left(t^{\prime}\right) b_{i}\left(t^{\prime}\right)-\left[d_{i}\left(t^{\prime}\right)+\frac{1}{2} \sigma_{i}\left(t^{\prime}\right)^{2}\right]\right\}
$$

and $U(t, 0)$ satisfies the evolution equation

$$
\mathscr{H}(t) u(t, 0) \equiv \sum_{i=1}^{3} \eta_{i}(t) \hat{e}_{i} u(t, 0)=\frac{\partial}{\partial t} u(t, 0), \quad \boldsymbol{U}(0,0)=1
$$


with

$$
\begin{array}{rlrl}
\eta_{1}(t) & =\left[\kappa(t) \theta(t)+2 c_{2}(t)\right] \exp \left[c_{1}(t)\right], \\
\eta_{2}(t) & =-\exp \left[-c_{1}(t)\right], & & \eta_{3}(t)=0, \\
\hat{e}_{1} & =\frac{\partial}{\partial r}, \quad \hat{e}_{2}=r, & \hat{e}_{3}=1 .
\end{array}
$$

The operators $\hat{e}_{i}$ form the Heisenberg-Weyl Lie algebra

$$
\left[\hat{e}_{1}, \hat{e}_{2}\right]=\hat{e}_{3}, \quad\left[\hat{e}_{1}, \hat{e}_{3}\right]=\left[\hat{e}_{2}, \hat{e}_{3}\right]=0 .
$$

Following a similar procedure as shown above, the operator $u(t, 0)$ is found to be

$$
u(t, 0)=\exp \left[\mu_{2}(t) \hat{e}_{2}\right] \exp \left[\mu_{1}(t) \hat{e}_{1}\right] \exp \left[\mu_{3}(t) \hat{e}_{3}\right]
$$

with

$$
\mu_{1}(t)=\int_{0}^{t} d t^{\prime} \eta_{1}\left(t^{\prime}\right), \quad \mu_{2}(t)=\int_{0}^{t} d t^{\prime} \eta_{2}\left(t^{\prime}\right), \quad \mu_{3}(t)=\int_{0}^{t} d t^{\prime} \mu_{2}\left(t^{\prime}\right) \eta_{1}\left(t^{\prime}\right) .
$$

As a result, we have obtained the exact form of the desired time evolution operator $U(t, 0)$ of the pricing equation in (4.1). It is then straightforward to show that $P\left(x_{1}, x_{2}, \ldots, x_{n}, r, t\right)$ is given by

$$
\begin{aligned}
P\left(x_{1}, x_{2}, \ldots, x_{n}, r, t\right)= & \int_{-\infty}^{\infty} d y_{1} \int_{-\infty}^{\infty} d y_{2} \ldots \int_{-\infty}^{\infty} d y_{n} P\left(y_{1}, y_{2}, \ldots, y_{n}, r, 0\right) \\
& \times K\left(x_{1}, x_{2}, \ldots, x_{n}, t ; y_{1}, y_{2}, \ldots, y_{n}, 0 ; r\right)
\end{aligned}
$$

where

$$
\begin{aligned}
K\left(x_{1},\right. & \left.x_{2}, \ldots, x_{n}, t ; y_{1}, y_{2}, \ldots, y_{n}, 0 ; r\right) \\
= & \frac{1}{\sqrt{(4 \pi)^{n} \operatorname{det}(\mathbf{a})}} \exp \left\{\mu_{3}(t)+c_{2}(t) \mu_{2}(t)^{2} \exp \left[2 c_{1}(t)\right]+\mu_{2}(t) \exp \left[c_{1}(t)\right] r\right\} \\
\quad & \times \exp \left\{-\frac{1}{4} \sum_{i, j=1}^{n}\left(x_{i}-y_{i}+v_{i}\right)\left(\mathbf{a}^{-1}\right)_{i j}\left(x_{j}-y_{j}+v_{j}\right)\right\}
\end{aligned}
$$

is the propagator of the pricing equation in (4.1) and

$$
v_{i}(t)=b_{i}(t) r+\xi_{i}(t)+\mu_{2}(t) f_{i}(t) \exp \left[c_{1}(t)\right] .
$$

The matrix $\mathbf{a}(t)$ is the $n \times n$ matrix whose elements are given by $a_{i j}(t)$, and $\mathbf{a}^{-\mathbf{1}}(t)$ is its inverse. Furthermore, in terms of the riskless bond function $Q(r, t)$ of the Vasicek model with explicitly time-dependent parameters, we can easily rewrite the propagator $K\left(x_{1}, x_{2}, \ldots, x_{n}, t ; y_{1}, y_{2}, \ldots, y_{n}, 0 ; r\right)$ and $v_{i}(t)$ as follows:

$$
\begin{aligned}
& K\left(x_{1}, x_{2}, \ldots, x_{n}, t ; y_{1}, y_{2}, \ldots, y_{n}, 0 ; r\right) \\
& \quad=\frac{Q(r, t)}{\sqrt{(4 \pi)^{n} \operatorname{det}(\mathbf{a})}} \exp \left\{-\frac{1}{4} \sum_{i, j=1}^{n}\left(x_{i}-y_{i}+v_{i}\right)\left(\mathbf{a}^{-1}\right)_{i j}\left(x_{j}-y_{j}+v_{j}\right)\right\}
\end{aligned}
$$

and $v_{i}(t)=-\ln [Q(r, t)]-a_{i i}(t)-\int_{0}^{t} d t^{\prime} d_{i}\left(t^{\prime}\right)$. 
For illustration, we consider the evaluation of a European call option on the maximum of two assets $S_{1}$ and $S_{2}$ with a strike price of $K$. The payoff at expiry for such an option is $\max \left(\max \left(S_{1}, S_{2}\right)-K, 0\right)$. Then the option price $P\left(S_{1}, S_{2}, r, t\right)$ is given by

$$
P\left(S_{1}, S_{2}, r, t\right)=I_{1}+I_{2}+I_{3}-K Q(r, t)
$$

where

$$
\begin{aligned}
& I_{1}=S_{1} N_{2}\left(\theta_{1}, \phi_{1}, \rho_{1}\right) \frac{\exp \left(-\int_{0}^{t} d t^{\prime} d_{1}\left(t^{\prime}\right)\right)}{\sqrt{1+\chi_{1}^{2}}}, \\
& I_{2}=S_{2} N_{2}\left(\theta_{2}, \phi_{2}, \rho_{2}\right) \frac{\exp \left(-\int_{0}^{t} d t^{\prime} d_{2}\left(t^{\prime}\right)\right)}{\sqrt{1+\chi_{2}^{2}}}, \\
& I_{3}=K Q(r, t) N_{2}\left(\theta_{3}, \phi_{3}, \rho_{3}\right) \text {, } \\
& \chi_{1}=\frac{a_{11}-a_{12}}{\sqrt{\operatorname{det}(\mathbf{a})}}, \quad \chi_{2}=\frac{a_{22}-a_{12}}{\sqrt{\operatorname{det}(\mathbf{a})}}, \\
& \rho_{1}=\frac{\chi_{1}}{\sqrt{1+\chi_{1}^{2}}}, \quad \rho_{2}=\frac{\chi_{2}}{\sqrt{1+\chi_{2}^{2}}}, \quad \rho_{3}=\frac{a_{12}}{\sqrt{a_{11} a_{22}}}, \\
& \theta_{1}=-\sqrt{\frac{\left(1-\rho_{1}^{2}\right)\left(a_{11}-2 a_{12}+a_{22}\right)}{2 \cdot \operatorname{det}(\mathbf{a})}} \cdot\left\{\ln \left(\frac{K Q}{S_{1}}\right)-a_{11}+\int_{0}^{t} d t^{\prime} d_{1}\left(t^{\prime}\right)\right\}, \\
& \phi_{1}=\sqrt{\frac{\left(1-\rho_{1}^{2}\right) a_{11}}{2 \cdot \operatorname{det}(\mathbf{a})}} \cdot\left\{\ln \left(\frac{S_{1}}{S_{2}}\right)+a_{11}-2 a_{12}+a_{22}-\int_{0}^{t} d t^{\prime} d_{1}\left(t^{\prime}\right)+\int_{0}^{t} d t^{\prime} d_{2}\left(t^{\prime}\right)\right\} \text {, } \\
& \theta_{2}=-\sqrt{\frac{\left(1-\rho_{2}^{2}\right)\left(a_{11}-2 a_{12}+a_{22}\right)}{2 \cdot \operatorname{det}(\mathbf{a})}} \cdot\left\{\ln \left(\frac{K Q}{S_{2}}\right)-a_{22}+\int_{0}^{t} d t^{\prime} d_{2}\left(t^{\prime}\right)\right\} \text {, } \\
& \phi_{2}=\sqrt{\frac{\left(1-\rho_{2}^{2}\right) a_{22}}{2 \cdot \operatorname{det}(\mathbf{a})}} \cdot\left\{\ln \left(\frac{S_{2}}{S_{1}}\right)+a_{11}-2 a_{12}+a_{22}-\int_{0}^{t} d t^{\prime} d_{2}\left(t^{\prime}\right)+\int_{0}^{t} d t^{\prime} d_{1}\left(t^{\prime}\right)\right\} \text {, } \\
& \theta_{3}=\frac{\ln \left(K Q / S_{1}\right)+a_{11}+\int_{0}^{t} d t^{\prime} d_{1}\left(t^{\prime}\right)}{\sqrt{2 a_{11}}}, \\
& \phi_{3}=\frac{\ln \left(K Q / S_{2}\right)+a_{22}+\int_{0}^{t} d t^{\prime} d_{2}\left(t^{\prime}\right)}{\sqrt{2 a_{22}}} .
\end{aligned}
$$

Here, $N_{2}(\theta, \phi, \rho)$ stands for the bivariate cumulative normal density function. It should be noted that, by setting $\sigma_{r}(t)=\rho_{1 r}(t)=\rho_{2 r}(t)=\kappa(t)=0$ in the above price function, we will obtain the option price $P\left(S_{1}, S_{2}, t\right)$ for the special case with nonstochastic short-term interest rate. Furthermore, as far as we know, the results in (4.26) and (4.27) are completely new. 
5. Conclusion. In this paper, we apply the Lie algebraic approach to valuation of multi-asset financial derivatives with time-dependent parameters. Based upon the dynamical symmetry of the pricing partial differential equations of the financial derivatives, the method is able to derive analytical closed-form pricing formulae very straightforwardly. We believe that the new approach will provide an efficient and easy-to-use method for the valuation of financial derivatives. Furthermore, this simple Lie algebraic approach can be easily extended to other financial derivatives with well-defined algebraic structures.

ACKNOWLEDGMENTS. This work was partially supported by the Direct Grant for Research from the Research Grants Council of the Hong Kong Government. The conclusions herein do not represent the views of the Hong Kong Monetary Authority.

\section{REFERENCES}

[1] L. P. Bos and A. F. Ware, Solving multi-asset Black-Scholes with time-dependent volatility, working paper, Mathematical and Computational Finance Laboratory, University of Calgary, Canada, 2000.

[2] C. F. Lo, Coherent-state propagator of the generalized time-dependent parametric oscillator, Europhys. Lett. 24 (1993), no. 5, 319-323.

[3] _ Propagator of the general driven time-dependent oscillator, Phys. Rev. A 47 (1993), 115-118.

[4] (_ Propagator of the Fokker-Planck equation with a linear force-Lie-algebraic approach, Europhys. Lett. 39 (1997), 263-267.

[5]_ Lie-algebraic approach for the generalized Fokker-Planck equation with a linear force, Nuovo Cimento Soc. Ital. Fis. B (12) 113 (1998), no. 12, 1533-1536.

[6] _ Propagator of the n-dimensional generalization of the Fokker-Planck equation with a linear force: Lie-algebraic approach, Phys. Lett. A 246 (1998), no. 1-2, 66-70.

[7] Lie-algebraic approach for the Fokker-Planck equation with a nonlinear drift force, Phys. A 262 (1999), no. 1-2, 153-157.

[8] C. F. Lo and C. H. Hui, Valuation of financial derivatives with time-dependent parameters: Lie-algebraic approach, Quant. Finance 1 (2001), 73-78.

[9] C. F. Lo and Y. J. Wong, Propagator of two coupled general driven time-dependent oscillators, Europhys. Lett. 32 (1995), 193-198.

[10] K. M. Ng and C. F. Lo, Coherent-state propagator of two coupled generalized timedependent parametric oscillators, Phys. Lett. A 230 (1997), no. 3-4, 144-152.

[11] O. A. Vasicek, An equilibrium characterization of the term structure, Journal of Financial Economics 5 (1997), 177-188.

[12] J. Wei and E. Norman, Lie algebraic solution of linear differential equations, J. Mathematical Phys. 4 (1963), 575-581.

C. F. Lo: Department of Physics, The Chinese University of Hong Kong, Shatin, New TERRITORIES, HONG KONG

E-mail address: cflo@phy.cuhk.edu.hk

C. H. Hui: BAnking Policy Department, Hong Kong Monetary Authority, 30th Floor, 3 GARDEN ROAD, HONG KONG

E-mail address: Cho-Hoi_Hui@hkma.gov.hk 


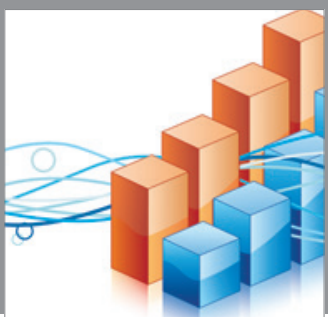

Advances in

Operations Research

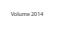

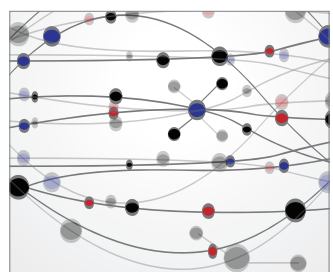

\section{The Scientific} World Journal
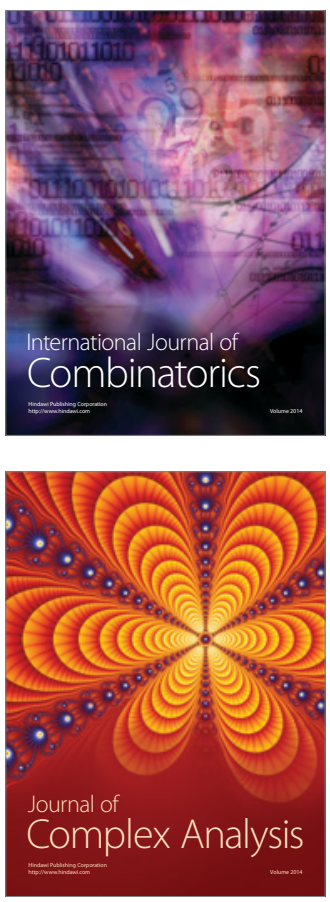

International Journal of

Mathematics and

Mathematical

Sciences
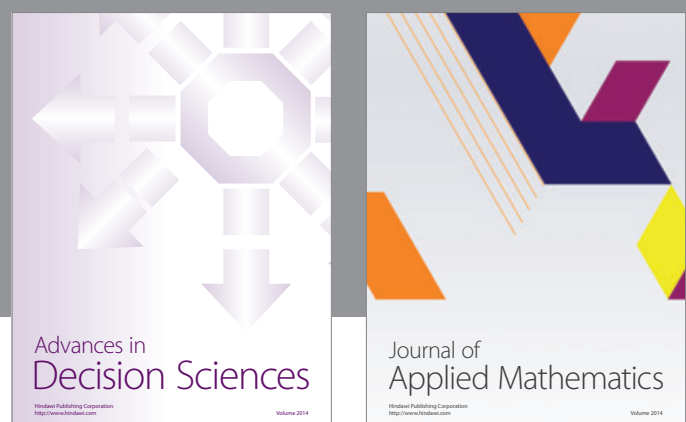

Journal of

Applied Mathematics
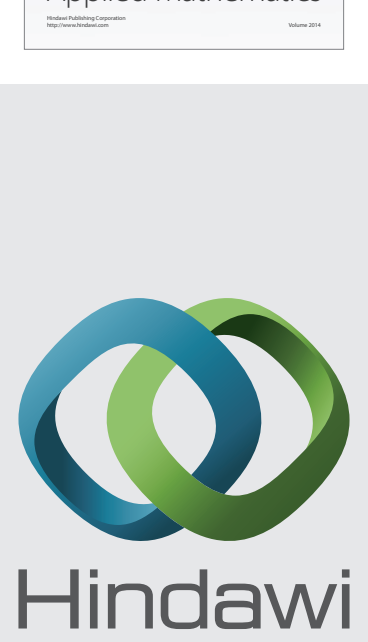

Submit your manuscripts at http://www.hindawi.com
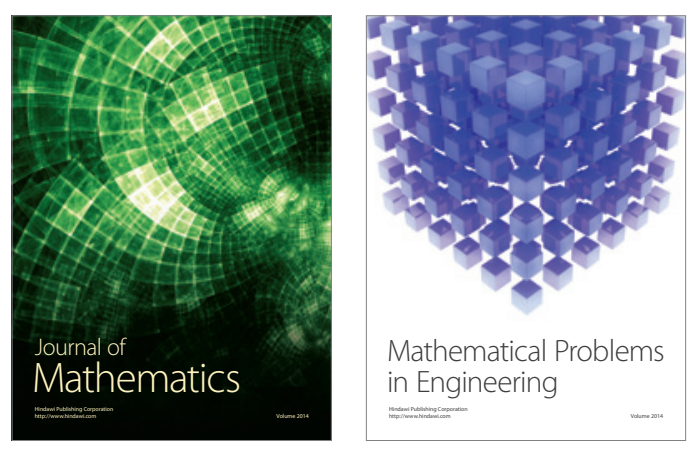

Mathematical Problems in Engineering
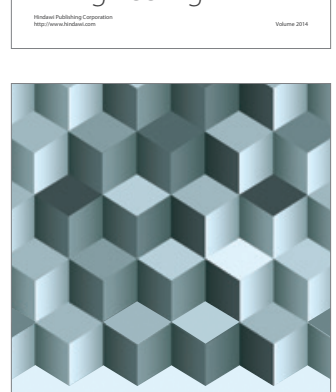

Journal of

Function Spaces
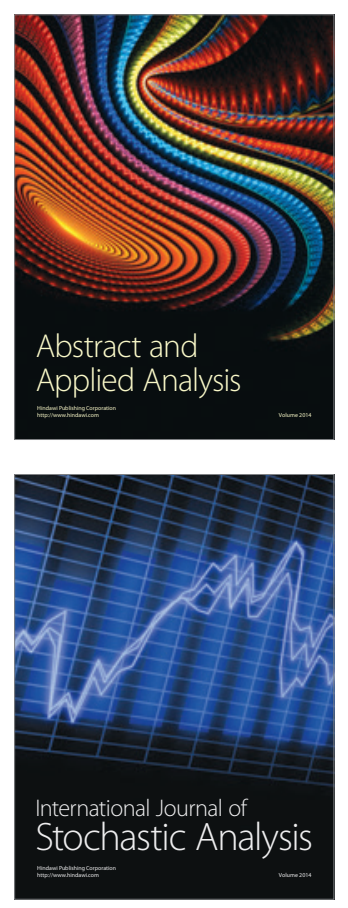

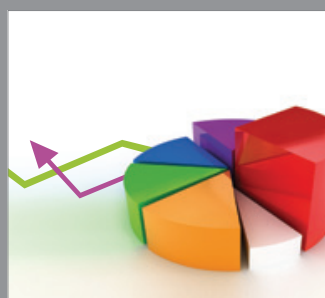

ournal of

Probability and Statistics

Promensencen
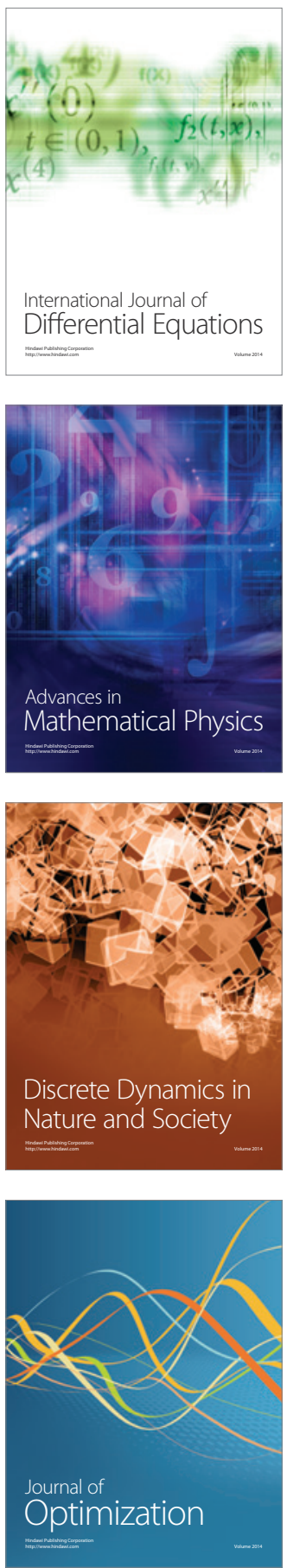